\section{Speeding Access to Precision Oncology Drugs: How Are We Doing With Biomarker-Driven Drug Approvals?}

Tianhong Li, MD, PhD

B iomarker-driven precision cancer therapy has revolutionized not only cancer diagnosis and treatment but also clinical trial design and FDA drug approval processes over the past 2 decades. ${ }^{1,2}$ Unlike the classic drug development pathway of progressing from phase I (maximum tolerated dose, dose-limiting toxicities, safety, and tolerability) to phase II (dose-finding, efficacy assessment), and then to phase III (randomized controlled trials designed to demonstrate efficacy and safety compared with standard of care in support of regulatory approval) for a single indication, which usually takes 10 to 15 years from first testing of a potential therapeutic agent to regulatory approval, ${ }^{3}$ each biomarker-driven clinical trial focuses on a specific genetic alteration in a small subset of targeted patients using response rate (RR) and duration of response (DoR) to measure clinical outcomes. This biomarker-driven clinical trial design has shortened the time from investigational new drug designation for novel agents to first FDA approval to approximately 4 years. In recent years, the FDA has approved an increasing number of precision oncology drugs targeted to individual molecular biomarkers across many cancer types based on early-stage (phase I or II) nonrandomized clinical trials. ${ }^{4}$

Several examples highlight the progress in FDA regulatory approval of biomarkerdriven precision oncology drugs. First, the small molecule tyrosine kinase inhibitor crizotinib received accelerated approval based on a specific genomic alteration (ALK gene rearrangement) in patients with advanced non-small cell lung cancer (NSCLC). ${ }^{5}$ Second, the clinical evaluation of pembrolizumab in the KEYNOTE-001 trial in patients with metastatic NSCLC led to the first breakthrough therapy designation in 2014 and subsequent accelerated FDA approval in 2015 for the treatment of patients with PD-L1-expressing metastatic NSCLC with disease progression on or after platinum-containing therapy. ${ }^{6}$ KEYNOTE-001 contained nested, multiple phase-II-like expansion cohorts of dose/schedule-comparison substudies in patients with melanoma and NSCLC, and training and validation sets for the development of the PD-L1 immunohistochemistry companion diagnostic assay. Each expansion cohort used the single-arm trial design as one of the accepted approaches for seeking accelerated approval. The use of biomarker-driven, multiple expansion cohorts allowed the simultaneous testing of multiple hypotheses (pertaining to populations, doses, and biomarker development) with appropriate type 1 error (false-positive) control in multiple tumor types. This design further expedited the development and approval of a promising novel drug based on early and strong efficacy signals in a phase I trial while retaining sufficient rigor to support regulatory filings. Third, in 2018, larotrectinib was approved in all solid tumors with NTRK gene fusions, rather than a specific tumor type. ${ }^{7}$ Approvals of these precision oncology drugs reflect the continuous effort from all stakeholders to bring new cancer therapeutics to the patients who need them. It is important to evaluate the use of appropriate clinical parameters in these studies that are considered during novel biomarker-driven precision oncology drug approval.

In this issue of JNCCN, Gyawali et al ${ }^{8}$ conducted a meta-epidemiologic analysis to determine the reliability of RR and DoR as endpoints in early-phase trials of

See page 36 for related article.

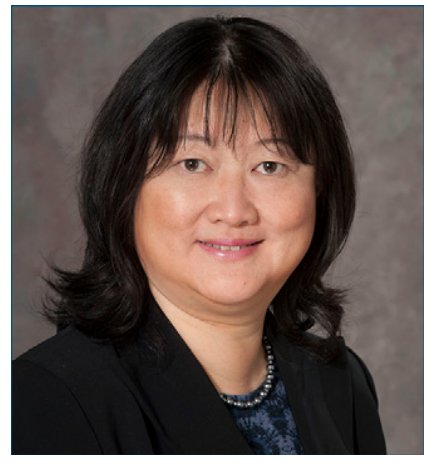

TIANHONG LI, MD, PhD

Tianhong $\mathrm{Li}, \mathrm{MD}, \mathrm{PhD}$, is an Associate Professor of Medicine in the Division of Hematology and Oncology, Department of Internal Medicine and the Experimental Therapeutics Program at the UC Davis Comprehensive Cancer Center. She obtained her MD degree from Peking University Health Science Center (formerly Beijing Medical University) and PhD in Molecular Immunology from Loyola University, Chicago. She pursued residency training in Internal Medicine at Beth Israel Medical Center, New York, and a Hematology/ Oncology fellowship at Montefiore Medical Center, Albert Einstein College of Medicine in the Bronx, New York. She is a boardcertified practicing medical oncologist specialized in experimental therapeutics focusing on lung and breast cancer. Her career goal is to translate new laboratory findings and technology innovations into direct patient care through early-phase clinical trials with correlative science studies.

doi: $10.6004 /$ jnccn.2019.7523

The ideas and viewpoints expressed in this commentary are those of the author and do not necessarily represent any policy, position, or program of NCCN. 
precision oncology drugs. Specifically, they identified 19 cancer drugs approved with biomarkers from the FDA's "Table of Pharmacogenomic Biomarkers in Drug Labeling"9 through December 2017. All the drugs have approved companion diagnostics except for nivolumab and pembrolizumab, for which consensus was not received for using PD-L1 expression as the biomarker. The investigators also excluded trials with different inclusion criteria in study subjects, line of treatment, and drug combination. They compared the RRs and DoRs of each drug between nonrandomized and randomized trials using similar design and subjects in the same cancer type. The ratio of $R R(r R R)$ or DoR ( $r D \circ R)$ was pooled across the trial pairs using random effects meta-analyses.

Gyawali et $\mathrm{al}^{8}$ found that the RR and DoR in nonrandomized trials were greater than those in randomized trials in most cases (ie, $63 \%$ and $87 \%$ of cases, respectively). The pooled rRR was $1.06(95 \% \mathrm{Cl}, 0.95-1.20)$ and the pooled rDoR was 1.17 (95\% Cl, 1.03-1.33). The slightly higher RR and DoR in the nonrandomized trials compared with those in the confirmatory randomized controlled trials could be because the trials were conducted in different patient populations or different regimens. They thus concluded that RR but not DoR is comparable between nonrandomized and randomized FDA-approved trials.

The study has several clinical implications. It was well done and provided important information supporting the FDA's accelerated drug approval programs, which has provided patients with cancer early access to promising, effective cancer therapeutics since its inception. The study also calls for further research in addressing the gaps and unmet needs in current drug development and regulatory sciences, because additional questions remain. First, is RR an immediate endpoint for meaningful clinical benefit? RR is an objective measurement of tumor shrinkage that has good correlation in nonrandomized and randomized clinical trials. ${ }^{8}$ Although RR might not be durable and result in survival benefits, it can be important in providing a rapid reduction in tumor burden, which palliates cancer-related symptoms, improves patient performance status, and enables patients to bridge to more effective but more toxic treatment.

Second, are there better biomarkers for clinical benefit? Recently, Osgood et a $\mathrm{a}^{10}$ from the FDA showed that depth of response ( $>75 \%$ as defined by RECIST) correlated with longer progression-free and overall survivals regardless of therapy type in 3,778 patients with unresectable or metastatic melanoma treated with targeted kinase inhibitors or immunotherapies in 10 randomized trials. However, analysis of depth of tumor response required knowing individual patients' response data that are not currently reported in most clinical trials. It will also be necessary to investigate the validity of this novel response marker for correlation with overall survival in other tumor types.

Third, are RR and DoR good biomarkers for evaluating cancer immunotherapy? The study by Gyawali et $a^{8}$ did not have enough cancer immunotherapy trials for evaluation. This could be the subject of future evaluation.

Finally, could other novel biomarkers be used to predict clinical benefit from precision oncology drugs? For example, measurement of minimal residual disease using liquid biopsy and circulating tumor biomarkers (such as DNA) is promising in quantifying treatment effect and disease control. Further studies are warranted to define the role of minimally invasive biomarkers in precision oncology drug approval trials.

In summary, the study by Gyawali et a $\left.\right|^{8}$ provides timely evaluation of the validity of objective RRs and DoRs as clinical biomarkers for overall survival using FDA-approved, biomarker-driven, early-phase clinical trials. Further studies are needed to explore innovative clinical evaluation of biomarker-driven precision cancer therapeutics to improve patient outcomes.

Disclosure: Dr. Li has disclosed that she is a scientific advisor for Foundation Medicine and ArcherDx, Inc., and receives grant/research support from Pfizer, LabyRx Immunologic Therapeutics Limited, Hengrui, and Merck.

Correspondence: Tianhong Li, MD, PhD, University of California Davis Comprehensive Cancer Center, 4501 X Street, Suite 3016, Sacramento, CA 95817. Email: thli@ucdavis.edu

\section{References}

1. Schwaederle M, Zhao M, Lee JJ, et al. Association of biomarker-based treatment strategies with response rates and progression-free survival in refractory malignant neoplasms: a meta-analysis. JAMA Oncol 2016;2:1452-1459.

2. Chakiba C, Grellety T, Bellera C, Italiano A. Encouraging trends in modern phase 1 oncology trials. N Engl J Med 2018;378:2242-2243.

3. Gandara DR, Li T, Lara PN Jr, et al. Algorithm for codevelopment of new drug-predictive biomarker combinations: accounting for inter- and intrapatient tumor heterogeneity. Clin Lung Cancer 2012;13:321-325.

4. Diabetes Patient Advocacy Coalition. How the FDA drug approval process works. Available at: https://diabetespac.org/fda-drug-approvalprocess/. Accessed December 1, 2019.

5. Selaru $P$, Tang $Y$, Huang $B$, et al. Sufficiency of single-arm studies to support registration of targeted agents in molecularly selected patients with cancer: lessons from the clinical development of crizotinib. Clin Transl Sci 2016;9:63-73.

6. Kang SP, Gergich K, Lubiniecki GM, et al. Pembrolizumab KEYNOTE-001: an adaptive study leading to accelerated approval for two indications and a companion diagnostic. Ann Oncol 2017;28:1388-1398.

7. FDA approves Vitrakvi (larotrectinib), the first ever TRK inhibitor, for patients with advanced solid tumors harboring an NTRK gene fusion. Available at: https://www.prnewswire.com/news-releases/ fda-approves-vitrakvi-larotrectinib-the-first-ever-trk-inhibitor-forpatients-with-advanced-solid-tumors-harboring-an-ntrk-gene-fusion 12-300755551.html. Accessed December 1, 2019.

8. Gyawali B, D'Andrea E, Franklin JM, Kesselheim AS. Response rates and durations of response for biomarker-based cancer drugs in nonrandomized versus randomized trials. J Natl Compr Canc Netw 2020;18: 36-43.

9. US Food and Drug Administration. Table of Pharmacogenomic Biomarkers in Drug Labeling. Available at: https://www.fda.gov/drugs/ scienceresearch/ucm572698.htm. Accessed December 9, 2019.

10. Osgood C, Mulkey F, Mishra-Kalyani PS, et al. FDA analysis of depth of response (DpR) and survival across 10 randomized controlled trials in patients with previously untreated unresectable or metastatic melanoma (UMM) by therapy type [abstract]. J Clin Oncol 2019; 37(Suppl):Abstract 9508. 